\title{
A Survey on Security Attacks/Defenses in Mobile Ad- hoc Networks
}

\author{
Ola $\mathrm{H}$. Younis \\ Computer Science \& Eng. \\ Dept., \\ Faculty of Electronic Eng., \\ Menoufia University, Menouf \\ 32952, Egypt
}

\author{
Salah E. Essa \\ Computer Science \& Eng. \\ Dept., \\ Faculty of Electronic Eng., \\ Menoufia University, Menouf \\ 32952, Egypt
}

\author{
Ayman EL-Sayed \\ Computer Science \& Eng. \\ Dept., \\ Faculty of Electronic Eng., \\ Menoufia University, Menouf \\ 32952, Egypt
}

\begin{abstract}
Security In mobile ad-hoc network (MANET) is a testing issue because of its unfastened characteristics, the feature of infrastructure less and nodes versatility. In outlining another security system for versatile Ad-hoc networks, one must consider the assaults varieties and in addition the qualities of the assaults that could be propelled facing the Ad-hoc networks and existing identification and moderation schemes. The analysis of these perspectives are outlined in this paper. A variety of attacks have been analyzed in Ad-hoc networks and also the proposed defenses against them. A short prologue to the sorts of assaults and conceivable counter measures to forestall or beat the assaults will be presented.
\end{abstract}

\section{Keywords}

MANET, Security, attacks, blackhole, wormhole, Challenges.

\section{INTRODUCTION}

A Mobile Ad-hoc Network is a [1] gathering of autonomous portable nodes that is shaped without the support of any current network structure. The MANET is a self-configurable network, where the nodes associate or detach from alternate nodes naturally at any time. The node to node availability and so on. Directing of the information are done on the premise of the node disclosure i.e. the node acknowledges the information and advances it to neighboring node in the path for the further sending with the goal that it can be come to the specific receiver. Every node act as a middle agent to complete the traffic of the information. As Dynamicity is the nature of MANET so it is available to every one of the clients it might be an honest to goodness client or the malevolent node which imitate the information or assault in the network.

The features of any Mobile Ad-hoc network is as follow: Distributed operation: background network does not exist to main control of the operation of the network, the management of the network is joint between the nodes. The nodes deployed in a MANET ought to coordinate with each other and convey among themselves and every node goes about as a hand-off as required, to actualize particular scope, as an example, directing and security, Multi hop routing: though a node tries to send data to different nodes which is out of its correspondence run, the packet ought to be sent by means of at least one halfway nodes, Autonomous terminal: each movable node is an autonomous node that can work as both a router and a host, In Dynamic topology: In this topology nodes are admitted to move subjectively with various rates; therefore, the network topology may change haphazardly and at eccentric time. In the MANET the nodes powerfully build up routing among each other as they go around, setting up their own network, Light-weight terminals: In extreme cases, at MANET the nodes are versatile with less CPU ability, low power stockpiling and little memory measure, Shared Physical Medium: The wireless medium of communication is open to any element with the fitting gear and satisfactory assets. Appropriately, access to the channel can't be limited.

Mobile specially is picking up prevalence because of it has a dynamic network and less frameworks. Ad-hoc network can be built up any place where the nodes have availability with different nodes and can join and leave the network whenever [1]. The applications are as taken after: Military combat zone: Military gear now routinely contains some kind of PC hardware [57]. During Ad-hoc network networking, the military could take the upside of typical system innovation to keep up a data arrange among the vehicles, troopers and military base camp. Essentially, the methods of ad-hoc network originated from this domain, Emergency Services: Ad hoc can be utilized as a part of crisis operations, for example, hunt and safeguard, recuperation from calamities for e.g. Fire, surge, well of lava seismic tremor, emission and so forth, Commercial part: Ad hoc can be utilized as a part of crisis/save operations for normal cataclysms alleviation endeavors, e.g. in flame, surge, or quake. Save operations must happen where non-existing or harmed correspondences framework and quick sending of a communication network is required. Data is conveyed starting with one save colleague then onto the next, Local level: Ad-Hoc network can self-governingly connect a moment and transitory sight and multimedia system utilizing note pad PCs to spread and share data among members at a e.g. gathering or classroom. Different fitting nearby level application may be in home networks where gadgets can convey specifically to exchange data, Personal Area Network (PAN): Short-run MANET can improve the intercommunication between different mobile device, (for example, a mobile device, portable workstations, and wearable PCs) [57]. Customary wired links are supplanted 
with remote associations. MANET can likewise reach out to get to the Internet or different network by mechanisms for example wireless LAN, and Collaborative work: according to business circumstances, the requirement for community oriented computing may be more essential outside office situations than inside and where individuals do need outside gatherings to coordinate and trade data on a given venture. There are a few vital necessities to accomplish security in MANETs, which are examined as takes after. Availability: guarantees survivability in spite of Denial of Service (DoS) assaults, Authentication: empowers a node to guarantee the identity of the associate node it is speaking with, Non-impersonation: which mean nobody else can put on a show to be another approved part to take in any helpful data [15, 44], Confidentiality: guarantees that information ought to be open just to the planned party. No other node aside from sender and recipient node can read the data. This is executed through information encryption systems [5], Integrity [54]: it implies that advantages can be changed just by approved gatherings or just in approved way. Adjustment incorporates composing, evolving status, erasing and making. Uprightness guarantees that a message being exchanged is never undermined, Authorization: [54] this equity doles out various get to rights to various sorts of clients. For instance, a network management can be performed by system manager just, Non-repudiation: Guarantees that the sender and the recipient of a message can't deny that they have ever sent or got a message [49], Attacks using fabrication: [6] which imply that the Generation of false steering messages is named as fabrication messages. Such assaults are hard to recognize, Data Verification: once the sender is validated the getting node performs information confirmations to check whether the message contains the right or tainted data[62], Privacy: the individual data must be kept up against unapproved access[62], Anonymity: it implies all data that can be utilized to distinguish proprietor or current client of node ought to default be kept private and not be disseminated by node itself or the framework software[54], Resilience to assaults: [64]it is required to manage the system functionalities when a segment of nodes is traded off or obliterated, and Freshness: guarantees that malignant node does not resend already caught packets[64].

An assortment of assaults are conceivable in MANET. These security assaults in MANET can be generally characterized by the accompanying norms: Active or Passive [13] [14]: An active assault includes data intrusion, change, or manufacture, accordingly disturbing the ordinary usefulness of a MANET, while an passive assault acquires information traded in the network without upsetting the operation of the interchanges, Internal or External [17]: External assaults are done by nodes that don't have a place with the space of the network. Internal assaults are from traded off nodes, which are quite of the network. Internal assaults are more extreme when contrasted and external assaults since the insider knows profitable and mystery data, and has advantaged get to rights, Attacks on different layers of the Internet model: the assaults can be further arranged by the five layers of the Internet model. A few assaults can be propelled at multiple layers. What's more, talk about assaults as indicated by this arrangement in points of interest will be proposed later, Stealthy versus non-stealthy assaults: some security assaults utilize stealth [24], whereby the assailants attempt to conceal their activities from either a person who is observing the framework or an intrusion detection framework (IDS). Be that as it may, different assaults, for example, DoS can't be made stealthy, and Cryptography versus non-cryptography related assaults: a few assaults are non-cryptography related, and others are cryptographic original assaults.

An assortment of security systems have been created to counter malevolent assaults [25]. The security mechanisms can be ordered into: Reactive mechanism: An intrusion detection system which considered as a second line of protection, and Preventive mechanism: The traditional authentication and encryption schemes are established using cryptography, which contains digital signature, asymmetric, and symmetric cryptography considered as a first line of protection.

There ought to be evaluation matrices to decide the execution or how solid the security mechanism is which are [8]: Time delay: Any kind of assault prompts to time defer in a system. This may additionally prompt to dismissing/disposing of the demand by collector, Loss of data assaults: such as Black hole assault, black hole assault vindictive nodes pulls in activity by giving inaccurate routing data and drops every one of a few information and in addition control packets going through it. In such cases, finish or halfway data misfortune happens, Fully/Partial paralyzing the network: on account of Fabrication assault, alteration assault when the connection is broken or directing table of nodes are demolished with defective data then there is a probability of incapacitating the network [9], Compromise $Q o S$ : Assaults like burrowing or worm hole assault trade off the security of network. In similar situation packet is sent to a node which is at multihop separate through a passage and divert back to network [10]. In issues like this the other may get entire data about network in this manner QoS is influenced, and Misuse of services: while any node do a childish conduct it tends to abuse the service gave by MANET. Like expending transmission capacity and surge the network.

This paper is arranged as next: Section 2 represent the categorization of attacks for MANET and the defenses for each attack. The future trends and Open points will be discussed in section 3. Finally, The work will be concluded in section 4 .

\section{TAXONOMY OF SECURITY ATTACKS}

Now an argue in details for the Attacks on Different Layers of the Internet Model, which are classified according to the five layers of the Internet model will be presented.

\subsection{Attacks at Physical Layer}

Eavesdropping: which can likewise be characterized as interception and perusing of messages and discussions by unintended beneficiaries. The fundamental point of such assaults is to get the classified data that ought to be kept mystery amid the correspondence [11]. 
Jamming: Is an uncommon class of DoS (Denial of Service) assaults which are started by vindictive node in the wake of deciding the frequency of communication. Which also likewise keeps the gathering of true valid packets. [11]

Defenses- Spread spectrum technology, for example, direct sequence (DSSS) [12] or frequency hopping (FHSS) [12], could shape it hard to distinguish or stick signals. Frequency is changed with it in an arbitrary manner to make signal catch troublesome or spreads the vitality to a more extensive range so the transmission power is holed up behind the level of commotion. Directional antennas apparatuses can likewise be sent because of the way that the communication techniques can be intended to spread the signal vitality in space. FHSS: tweaking of the signal is with an apparently irregular arrangement of radio frequencies, which jump from frequency to frequency at settled interims. The beneficiary uses a similar spreading code, which is synchronized with the transmitter, to recombine the spread signals into their unique frame. DSSS: Every information bit in the first signal is spoken to by numerous bits in the signal of transmission, utilizing a spreading code. The spreading code spreads the signal over a more extensive frequency band in direct extent to the quantity of bits utilized. The beneficiary can utilize the spreading code with the signal to recoup the first data. Both FHSS and DSSS posture challenges for untouchables endeavoring to catch the signals of radio. The meddler must know the frequency band, spreading code, and adjustment strategies with a specific end goal to precisely read the transmitted signals. In spite of the capacity of spread range innovation, it is secure just when the hopping pattern or spreading code is obscure to the busybodies.

\subsection{Data Link / MAC Layer Attacks}

Selfish Misbehavior of Nodes: Assaults under this class, are straightforwardly influences the self-execution of nodes and does not meddle with the handiwork of network. It might incorporate two variables: battery power Conservation and increasing unreasonable share of data transfer capacity. The narrow minded nodes may decline to participate in the sending procedure or drops the packets purposefully. These assaults misuse the routing protocols to their own particular leverage. Packet losses is one of the principle assaults by selfish node which prompts to clog in network. However a large portion of routing protocols have no component to recognize whether the packet being sent or not with the exception of DSR (dynamic source routing) [11].

Malicious Behavior of Nodes: The fundamental assignment of pernicious node is to routing protocol disturb ordinary operation. The effect of such assault is expanded when the communication happens between neighboring nodes. Assaults of such sort are fall into taking after classifications: Attacks on Network integrity, Misdirecting movement, and Denial of Service (DOS).

Traffic Analysis: In this sort of assault the enemies break down the traffic patterns to increase vital data on network topology that thus uncovers the data about the nodes. Data, for example, area of nodes, network topology used to impart and parts played by the nodes can be assembled.
Defenses- To ensure against Selfish Misbehavior of Nodes and malevolent actions of Nodes, Marti et al. [27] presented the idea of watchdog and path rater to enhance execution of ad-hoc network within the sight of troublesome or getting into mischief nodes. Watchdog duplicates packets to be sent into a cushion and screens the conduct of the nearby nodes to these packets. It wantonly eavesdrops the packets and if matches with the watching node's cradle, then they are disposed of. Path rater takes a shot at an individual node to rate all the neighboring nodes in its network concerning their reliabilities based upon the data go by the watchdog. Every node begins with a nonpartisan rating which is changed amid packet routing relying on their conduct and unwavering quality. Bad behavior and trickiness of nodes are recognized independently from each other. The avoidance of traffic analysis is by encryption at the interface layer of data link. WEP has been broadly scrutinized. An active blend technique is utilized to conceal the source and goal data amid message conveyance by means of cryptography strategy and to "blend" the network nodes [27]. WEP and WPA gives verification component to any node to network participate. LLSP is utilized to give security at data link layer. Be that as it may, LLSP utilizes encryption calculation to keep from assaults. SLSP is utilized to avert DOS assault, Man in the middle assault and it's appropriate for validating new nodes and not reasonable for ongoing movement.

\subsection{Network Layer Attacks}

Black Hole Attack: A vindictive node can infuse or embed false route answers to the route asks for it gets, publicizing itself as having the most limited path to a goal [15].

GrayHole Attacks: is uncommon variety of black hole assault. In black hole assault the aggressor places itself in the middle of the source. The aggressor pulls in the information packets to it by publicizing itself having the briefest route to receiver and after that they catch the information packet and drops it. In grayhole assault the information packets are dropped specifically or in measurable way. For example, they may drop packet from a specific node or in some other shape [30].

Jellyfish Attack: In this assault a noxious node makes nonsensical postponement for every one of the packets that were gotten for some measure of time before sending it [20]. Jellyfish assailant expects to expand end-to-end defer and make high deferral, jitter, which influences the ordinary execution of the network. Clearly, this is a refusal of administration assault.

Wormhole Attacks: an aggressor register packets at one area in the network and passages them to another area. Routing could be confused while routing control messages are burrowed. This passage between two intriguing assailants is alluded as a wormhole [31] [41]. Wormhole assaults are extreme dangers to MANET routing protocols.

Rushing Attack: There are some on-request routing protocols that utilization the copy concealment method. In this system amid the routing uncovering handle if any node gets a similar route request for packet (RREQ) more than one time, it consequently disposes of this copy 
packet. The assaulting node abuses this helpless procedure by flooding the system rapidly by the route inquiry for it gets to achieve the goal before a similar route request for achieves the goal through different nodes. Subsequently, the goal will dispose of the later honest to goodness inquiry for instead of process it [28].

Cache Poisoning Attack: commonly, in AODV, every node preserve few of its latest transmission route until timeout happens for every section. In this way, every route waits for quite a while in node's memory. On the off chance that some malevolent node plays out a directing assault, then they will remain in node's route table until timeout happens or a superior route is found. An assailant node can publicize a zero metric to the majority of its goals. Such route won't be overwritten unless timeout happens. It can even promote itself as a route to a removed node which is out of its compass. When it turns into a piece of the route, the assailant node can play out its malevolent action [32].

Location Disclosure Attack: An assailant finds the Location of a node or structure of whole network and unveil the security necessity of network. The enemy attempt to make sense of the correspondence parties and examine movement to take in the network activity design. The spillage of such data is dangerous for security.

Sybil Attack: It alludes to the many duplicates of malignant nodes. It can be happened, if the malevolent node conveys its secret key with different vindictive nodes. Along these lines the quantity of malignant node is expanded in the network and the likelihood of the assault is likewise expanded [35].

Neighbor Discovery Attack: Assault across Bluetooth in a blue tooth structure is all around clarified in [39]. Shortcoming in conventions can be misused to perform malignant neighbor disclosure. An assailant for this situation compels a casualty node to uncover private information, for example, its identification.

Packet Drop Attack: Vindictive or aggressor nodes loss all packets that are not bound for them. Malevolent nodes expect to upset the network association and execution, while narrow minded nodes plan to save their assets. The attack of packet dropping can avert end-to-end correspondences between nodes, if the dropping node is at a basic point. It may likewise decrease the system execution by retransmitting the packet of data.

Impersonation Attack: In impersonation assault aggressor nodes mimics itself as honest to goodness node and sends false routing data and covers itself as sending from trusted node [40].

Modification Attack: Modification incorporates composing, changing status and erasing from information packets in an unapproved way by the noxious nodes that take an interest in the packet sending process [43] [25] [45]. This kind of assault jeopardizes obviously the honesty of the network packets.

Byzantine Attack: In this assault at least one bargained nodes cooperates to make loops in routing path or such nodes advances the packet on the non-ideal paths subsequently influencing the QoS.
Defenses- There are two guard ways to ensure against BlackHole Attack. First of all, gathering different RREP messages (from more than two nodes) and consequently trusting numerous excess ways to the goal node and after that buffering the packet until a sheltered rout is raise. After that, keeping up a table in every node with past arrangement number in expanding request. Every node before sending packet expands the arrangement number. The sender node broadcast RREQ to its neighbors and once this RREQ achieves the goal, it answers with a RREQ with last packet grouping number. On the off chance that the halfway node finds that RREQ contains a wrong arrangement number, it comprehends that some place something turned out badly [16].

Priority protocol schemes are used to GrayHole Attacks. At whatever point a node enters in a Mobile Ad Hoc network IP assignment is the initial phase in which the node will get its IP alongside starting need and the method has been received in DHCP [18]. The second step is Neighbor Discovery of the presented scheme. New node will send the HELLO bundles to its neighbors and find the personality of the neighbors alongside their need. Confirmation is the following stride of the plan in which it will communicate data about its reality and trade keys with the neighbors as per the plan HEAP [19] which is a hopby-hop verification protocol. HEAP confirms packets at each jump by utilizing a changed HMAC based calculation alongside two keys and drops any packets that start from exterior. There are distinctive barriers against Jellyfish Attack The primary way is, 2ACK [21] The fundamental thought of the 2ACK plan is that, when a node advances an information packet effectively throughout the following bounce, the goal node of the following jump connection will send back an uncommon two-bounce affirmation called $2 \mathrm{ACK}$ to show that the information packet has been gotten effectively. Such a $2 \mathrm{ACK}$ transmission happens for just a small amount of information packets, yet not for all. The second way is Credit based system [16], this way gives impetuses to effective transmission or some likeness thereof of token or credit which the node may utilize when it begins sending its own particular bundle. Reputation based scheme: Here individual nodes by and large distinguish making trouble nodes, (for example, CONFIDANT) [22] [23]. A defense against the Wormhole assault is packet leash protocol [42]. The SECTOR technique [46] is presented to recognize wormholes without needing of clock synchronization. Directional antennas apparatuses [47] are likewise projected to counteract wormhole assaults. To keep the Rushing Attack, Two components could be utilized together which are, randomized route request forwarding and secure route delegation. Source routing delegation system is utilized to check that all the safe neighbor identification method are performed between two neighboring nodes. Randomized message sending arbitrary choice procedure can be utilized to keep the surging aggressors in overwhelming every other route to goal. Two parameters are utilized for determination of randomized sending they are the quantity of demand bundles to be gathered and calculation which can pick timeouts [28]. To avert Cache Poisoning Attack, SAODV [33] could be used: Secure AODV is an expansion to AODV convention that adds every node to trade marked route messages. Every node has its own open 
key which it uses to sign route messages. Likewise, SAODV utilizes hop count as a metric for most limited routes as AODV and utilizations hash chains to secure hop include data route messages. Additionally SNRP could be utilized [34]: Secure Neighbor Routing protocol utilizes security upgraded Neighbor Lookup Protocol (NLP) to secure MANET routing. Recently added node utilizes open key to take part in MANET. For guarding Location Disclosure Attack a technique utilizes geometric imperatives and heuristics [63] to discover node positions productively can be utilized to forestall such assault. In view of the restriction exactness that such an "omniscient" aggressor can achieve, the nature of future will has the capacity to be assessed, more reasonable assault models. One method for moderating Sybil Attack is keeping up a chain of trust, so single personality is produced by a various leveled structure which might be difficult to fake. Another approach would be founded on signal strength [36] [37] [38]. Approval procedures can be utilized to anticipate Sybil assaults and expel disguising unfriendly elements. A nearby element may acknowledge a remote personality in light of a central authority which guarantees a coordinated correspondence between a character and a substance and may even give an invert query. A personality might be approved either straightforwardly or by implication. In direct approval, the neighborhood element questions the central authority to approve the remote characters. In aberrant approval, the nearby substance depends on officially acknowledged characters which thus vouch for the legitimacy of the remote personality being referred to. Character based approval methods for the most part give responsibility to the detriment of secrecy, which can be an undesirable tradeoff particularly in online gatherings that desire to allow oversight free data trade and open exchange of touchy points. [3]. Many arrangements For Neighbor Discovery Attack that depend on the operators of home system are presented yet issue has not been illuminated yet. The author in [39] well clarifies how a blue tooth system casualty is seen by an arrangement of assailants in the system. Answer for this is if the personality of a gadget changes for every session it gets to be distinctly troublesome for an assailant to follow the area of the casualty. Certainly the complexity of addressing schemes is raised. For the attack of Packet Drop there are a two collapsed approach, to recognize and after that to confine such nodes is presented which turns into the piece of the network to bring about packets dropping assaults [61]. To protect against Impersonation Attack a multifaceted validation network is suggested that amplifies the cryptographic connection, restricting a substance to a physical node gadget. ARAN [41] can be utilized to shield against repudiation and impersonation assaults. SEAD [29] is utilized here for instance of a protection against Modification Attacks. Like a packet leash, in the protocol SEAD one way hash function is used to keep malevolent nodes from expanding the arrangement number or diminishing the jump tally in the packets of route advertisement. Another key management scheme [26] is executed in NTP protocol could likewise be an answer for this assault, since Node Transition Probability (NTP) based calculation gives most extreme use of data transmission amid substantial movement with less overhead. Different security plans are examined in [48] against Byzantine assault. It incorporates channel mindful location calculation which recognizes particular sending, hash function based strategy which creates behavioral confirmations in view of information movement and sending ways, DCIID calculation utilizing packet verifiers, Cooperative discovery component and so forth. IDS is presented in [4] for recognizing byzantine assault in AODV. In this plan, IDS screen the network figure before the section of node and after the node termination. On the off chance that profile change is distinguished by IDS, it is dealt with as assault. An amusement hypothesis [50] is likewise presented in assault resistance framework to distinguish byzantine assault. This hypothesis is of extraordinary help inside the environment with substantial number nodes.

\subsection{Transport Layer Attacks}

SYN flooding Attack: the assailant makes a substantial number of half opened TCP connection with a casualty node, however never finishes the handshake to completely open the connection. Amid the assault, a vindictive node sends a lot of SYN packets to a casualty node, mocking the arrival locations of the packet of SYN [51].

Session hijacking: The assailant parodies the casualty's IP address, decides the right succession number that is normal by the objective, and afterward plays out a DoS assault on the casualty. Consequently, the aggressor mimics the casualty node and proceeds with the session with the objective [51].

Masquerading: Amid the process of neighbor acquisition, an outside gatecrasher could disguise a nonexistent or present IS by appending itself to correspondence interface and wrongfully participating in the routing protocol area by bargaining verification framework. The risk of disguising is practically the same as that of a bargained IS [52].

Man-in-the-middle attacks: An aggressor sits between the sender and the collector and sniffs any data being sent between two closures. Now and again, assailant may mimic the sender to speak with recipient or imitate the collector to answer to the transmitter [53].

Replay Attack: An assailant that plays out a replay assault are retransmitted the legitimate information over and over to infuse the system routing activity that has been caught already. This assault more often than not focuses on the freshness of routes, yet can likewise be utilized to undermine inadequately composed security arrangements [54].

Defenses- Session Hijacking Point-to-point or end-to-end encryption, and SYN Flooding Attack gives message privacy over the transport layer in two end frameworks. Transport layer contains a connection-oriented reliable protocol which called TCP. Since TCP does not perform well in MANET, TCP feedback (TCP-F) [55], TCP explicit failure notification (TCP-ELFN) [55], ad hoc transmission control protocol (ATCP) [55], and ad hoc transport protocol (ATP) [55] have been imagined, yet none of these conventions are outlined because of security. Private Communications Transport (PCT) [56], Transport Layer Security (TLS) [56], Secure Socket Layer (SSL) [56], and protocols were designed for making the 
communication secured and are relayed on public key cryptography. The Attacks of Masquerading, and Man-inthe-middle: TLS/SSL can help secure information transmission, it can likewise ensure against these assaults TLS/SSL depends on public key cryptography, which is CPU-concentrated and requires far reaching regulatory design. Hence, the use of these plans in MANET is limited. TLS/SSL must be adjusted with a specific end goal to address the exceptional needs of MANET. A few firewall at a larger amount can be arranged to protect against the assaults. An answer has been proposed by Some Researcher for shield a MANET from a Replay Attack by utilizing a time stamp with the utilization of an asymmetric key. This arrangement keeps the replay assault by looking at the present time and time stamp contained in the got message. On the off chance that the time stamp is too a long way from the present time, the message is judged to be suspicious and is rejected [61].

\subsection{Application Layer Attacks}

There are many protocols supported in the application layer, for example, HTTP, SMTP, and FTP, it also contains the user data. Malignant Code Attacks: Malicious code assaults incorporate Viruses, Worms can influence both working framework and client application [2]. Renouncement/Repudiation Attacks: Repudiation alludes to a disavowal of interest in all or some portion of the interchanges. For instance, an egotistical node can prevent the preparing from securing an online bank exchange [2].

Defenses- Like any other layer, the application layer likewise should be secured against Malicious Code Attacks and Repudiation Attacks. In a system with a firewall introduced, the firewall can give get to control, client validation, packet separating, and a logging and bookkeeping administration. Application layer firewalls can viably anticipate many assaults, and applicationparticular modules, for instance, spyware identification programming, have additionally been created to defense mission-basic services. Be that as it may, a firewall is generally limited to fundamental get to control and is not ready to tackle all security issues. For instance, it is not viable against assaults from insiders. As a result of MANET's absence of foundation, a firewall is not especially valuable. In MANET, an Intrusion Detection System (IDS) which can be used as a second protection line. Intrusion Detection can be introduced at the network layer, yet in the application layer it is doable, as well as vital [25].

\subsection{Multi-layer attacks}

Some security assaults can be propelled from various layers rather than a specific layer. Cases of multi-layer assaults are denial of service (DoS).

Denial of service: Denial of service (DoS) assaults could be propelled from a different layers. An aggressor can utilize signal jamming at the physical layer, which disturbs ordinary interchanges. At the link layer, pernicious nodes could possess channels using the capture effect, which exploits the double exponential plan in MAC protocols and keeps different nodes from channel get to.

Denial of service at the network layer: The routing layer Assaults might comprise of yet is not restricted to the accompanying mischievous activities [58]: 1) The pernicious node takes an interest in a route however essentially drops a portion of the information packets, 2) the noxious node transmits distorted route refreshes, 3 ) the vindictive node could conceivably replay stale updates, and 4) the malignant node diminishes the TTL (time-tolive) field in the IP header so that the packet never achieves the goal.

DoS at the MAC Layer: The DoS assaults at the MAC layer, could incorporate, among others, the accompanying mischievous activities [58]: Keeping the direct occupied in the region of a node prompts to a dissent of administration assault at that node, and by utilizing a specific node to ceaselessly hand-off spurious information, the battery life of that node might be depleted.

Defenses- If end-to-end validation is authorized in Denial of Service at the system layer, assaults by autonomous malignant node of sorts second and third might be upset. An assault of sort first might be dealt with by relegating certainty levels to nodes and utilizing routes that give the most elevated amount of certainty. The fourth assult might be countered by making it obligatory that a hand-off node guarantees that the TTL field is set to an esteem more prominent than the hop number to the expected goals. On the off chance that nodes intrigue, the verification systems come up short and it is an open issue to give security against such routing assaults. For protecting against the MAC Layer Denial of Service, End-to-end verification may keep the over two cases from succeeding. On the off chance that the node does not have an endorsement of verification, it might be kept from getting to the channel. Generally the nodes are outcasts. Be that as it may, if nodes connive, and the plotting nodes incorporate the sending node and the goal, MAC layer assaults are extremely achievable.

\section{DISCUSSION AND OPEN POINT}

As of not long ago a quickly talked about the security assaults in MANET and has presented accessible protections components on it. Notwithstanding numerous qualities, the MANET presents a few difficulties that must be examined precisely for scientists. These are taking after:

Routing: Because of the continually changing topology in ad-hoc networks, the packet routing between any match of nodes turns into a testing errand. The majority of the protocols in view of receptive routing rather than proactive routing. Multi cast routing is another test as the multi cast tree is not static because of the arbitrary development of nodes inside the system. Routes among the nodes may have numerous hops, which is more intricate than the single hop communication.

Quality of Service: Giving diverse nature of service levels in an always showing signs of change condition will be a test. The inborn stochastic normal for interchanges quality in a MANET makes it hard to offer settled certifications on the services offered to a gadget. A versatile Quality of Service must be executed over the conventional asset reservation to bolster the services of multimedia.

Inter-networking: Notwithstanding the correspondence inside an ad hoc network, inter-networking amongst 
MANET and framework systems (principally IP based) is regularly expected as a rule. The routing protocols concurrence in such a mobile device is a test for versatility management.

Multicast: It is alluring to bolster multiparty wireless interchanges. As the multicast tree is not static, the routing protocol must have the capacity to adapt to portability including multicast enrollment flow (leave and join). Each node goes about as a router and can send packets of information to different nodes to give data sharing among the portable nodes. Ad hoc addressing scheme is troublesome task to be implemented, the MAC address of the gadget is utilized as a part of the remaining solitary ad hoc network. Nonetheless, every application depends on TCP/IP and UDP/IP

Scalability: which is needed in MANET as it is utilized as a part of military correspondences, due to that the network becomes as per the need, so every mobile device must be proficient to deal with the increase of network and to fulfill the errand.

\section{CONCLUSION}

In this paper, a discussion about various sorts of security assaults in MANET is presented. Additionally, a concentration has proposed to diverse safety efforts recommended for location and counteractive action of few assaults in MANET like Blackhole assault, Grayhole assault, and Byzantine assault. Every one of the strategies overviewed for this paper have proposed few changes in the routing protocols. In light of this study it can be proposed as there is significantly more degree in the field of assault identification and anticipation schemes for MANET.

\section{REFERENCES}

[1] Jain, S., \& Hemrajani, N. 2013. Detection and mitigation techniques of black hole attack in MANET: An Overview. International Journal of Science and Research (IJSR), India Online ISSN, 2319-7064.

[2] Venna, S. R., \& Inampudi, R. B. 2016. A Survey on Security Attacks in Wireless Sensor Network. Int. J. Comput. Sci. Inf. Technol.

[3] Paramesvaran, R. D., \& Maheswari, D. 2016. Study of Various Security Attacks in Network Layer and the Mitigation Techniques for MANET. Int. J. Adv. Res. Comput. Commun. Eng.

[4] Agrawal, N., Joshi, K. K., \& Joshi, N. 2015. Implemented and Evaluated the Byzantine Attack with the Aid of Rushing Attack in Manet. International Journal of Computer Applications, 130(6), 6-11.

[5] Goyal, P., Parmar, V., \& Rishi, R. 2011. Manet: vulnerabilities, challenges, attacks, application. IJCEM International Journal of Computational Engineering \& Management, 11(2011), 32-37.

[6] Singh, K., \& Yadav, R. S. 2007. A review paper on ad hoc network security. International journal of computer science and security, 1(1), 52.
[7] Singh, T., Singh, J., \& Sharma, S. 2016. Survey of secure routing protocols in MANET. International Journal of Mobile Network Design and Innovation, 6(3), 142-155.

[8] Jadye, S. (2016). Survey of MANET Attacks, Security Concerns and Measures. (IJCSIT) International Journal of Computer Science and Information Technologies, 7 (2), 1014-1017.

[9] Dorri, A., Kamel, S. R., \& Kheirkhah, E. 2015. Security challenges in mobile ad hoc networks: A survey. arXiv preprint arXiv:1503.03233.

[10] Garg, A., \& Beniwal, V. 2012. A review on security issues of routing protocols in mobile ad-hoc networks. International Journal of Advanced Research in Computer Science and Software Engineering, 2(9).

[11] Kour, P., \& Panwar, L. C. 2014. A Review on Security Challenges and Attacks in Wireless Sensor Networks. International Journal of Science and Research, 3(5), 1360-1364.

[12] Stallings, W. 2009. Wireless communications \& networks. Pearson Education India.

[13] Yi, S., \& Kravets, R. 2004. Composite Key Management for Ad Hoc Networks. In MobiQuitous (Vol. 4, pp. 52-61).

[14] Oppliger, R. 2001. Internet and intranet security. Artech House.

[15] Ghaffari, A. 2006. Vulnerability and security of mobile ad hoc networks. In Proceedings of the 6th WSEAS International Conference on Simulation, Modelling and Optimization (pp. 124-129). World Scientific and Engineering Academy and Society (WSEAS).

[16] Shen, H., \& Li, Z. 2008. ARM: An account-based hierarchical reputation management system for wireless ad hoc networks. In Distributed Computing Systems Workshops, 2008. ICDCS'08. 28th International Conference on (pp. 370-375). IEEE.

[17] Cardenas, A. A., Benammar, N., Papageorgiou, G., \& Baras, J. S. 2004. Cross-layered security analysis of wireless ad hoc networks. Maryland Univ College Park Dept Of Electrical And Computer Engineering.

[18] Menaria, S., Valiveti, S., \& Kotecha, K. 2010. Comparative study of distributed intrusion detection in ad-hoc networks. International Journal of Computer Applications, 8(9), 11-16.

[19] Anjum, F., \& Mouchtaris, P. 2007. Security for wireless ad hoc networks. John Wiley \& Sons.

[20] Purohit, N., Sinha, R., \& Maurya, K. 2011. Simulation study of Black hole and Jellyfish attack on MANET using NS3. In Engineering (NUiCONE), 2011 Nirma University International Conference on (pp. 1-5). IEEE.

[21] Liu, Z., Joy, A. W., \& Thompson, R. A. 2004. A dynamic trust model for mobile ad hoc networks. 
In Distributed Computing Systems, 2004. FTDCS 2004. Proceedings. 10th IEEE International Workshop on Future Trends of (pp. 80-85). IEEE.

[22] Bhalaji, N., Sivaramkrishnan, A. R., Banerjee, S., Sundar, V., \& Shanmugam, A. 2009. Trust enhanced dynamic source routing protocol for adhoc networks. World Academy of Science, Engineering and Technology, 49, 1074-1079.

[23] Buchegger, S., \& Le Boudec, J. Y. 2002. Performance analysis of the CONFIDANT protocol. In Proceedings of the 3rd ACM international symposium on Mobile ad hoc networking \& computing (pp. 226-236). ACM.

[24] Jakobsson, M., Wetzel, S., \& Yener, B. 2003. Stealth attacks on ad-hoc wireless networks. In Vehicular Technology Conference, 2003. VTC 2003-Fall. 2003 IEEE 58th (Vol. 3, pp. 2103-2111). IEEE.

[25] Wu, B., Chen, J., Wu, J., \& Cardei, M. 2007. A survey of attacks and countermeasures in mobile ad hoc networks. In Wireless network security (pp. 103135). Springer US.

[26] Radha, S. 2010. A novel method for detection and elimination of modification attack and TTL attack in NTP based routing algorithm. In Recent Trends in Information, Telecommunication and Computing (ITC), 2010 International Conference on (pp. 60-64). IEEE.

[27] Marti, S., Giuli, T. J., Lai, K., \& Baker, M. 2000. Mitigating routing misbehavior in mobile ad hoc networks. In Proceedings of the 6th annual international conference on Mobile computing and networking (pp. 255-265). ACM.

[28] Hu, Y. C., Perrig, A., \& Johnson, D. B. 2003. Rushing attacks and defense in wireless ad hoc network routing protocols. In Proceedings of the 2nd ACM workshop on Wireless security (pp. 30-40). ACM.

[29] Hu, Y. C., Johnson, D. B., \& Perrig, A. 2003. SEAD: Secure efficient distance vector routing for mobile wireless ad hoc networks. Ad hoc networks, 1(1), 175-192.

[30] Nadeem, A., \& Howarth, M. P. 2013. A survey of manet intrusion detection \& prevention approaches for network layer attacks. IEEE Communications surveys and tutorials, 15(4), 2027-2045.

[31] Ilyas, M. (Ed.). 2002. The handbook of ad hoc wireless networks. CRC press.

[32] Kaur, J., \& Nagpal, S. 2014. Review Paper on Security Challenges and Attacks in Mobile Ad-Hoc Networks. International Journal of Advanced Research in Computer Science and Software Engineering, 4(5), 1501-1508.

[33] Zapata, M. G. 2002. Secure ad hoc on-demand distance vector routing. ACM SIGMOBILE Mobile Computing and Communications Review, 6(3), 106107.
[34] Jadhav, A., \& Johnson, E. E. 2006. Secure neighborhood routing protocol. In Military Communications Conference, 2006. MILCOM 2006. IEEE (pp. 1-7). IEEE.

[35] Balamurugan, G., Somasundaram, R., \& Sumithra, K. 2014. The Comparative Study of Security Mechanism in Mobile Ad-hoc Networks. IJCSN - Int. J. Comput. Sci. Network.

[36] Abbas, S., Merabti, M., \& Llewellyn-Jones, D. 2009. Signal strength based Sybil attack detection in wireless Ad Hoc networks. In Developments in eSystems Engineering (DESE), 2009 Second International Conference on (pp. 190-195). IEEE.

[37] Hashmi, S., \& Brooke, J. 2010. Towards sybil resistant authentication in mobile ad hoc networks. In Emerging Security Information Systems and Technologies (SECURWARE), 2010 Fourth International Conference on (pp. 17-24). IEEE.

[38] Guette, G., \& Ducourthial, B. 2007. On the Sybil attack detection in VANET. In Mobile Adhoc and Sensor Systems, 2007. MASS 2007. IEEE International Conference on (pp. 1-6). IEEE.

[39] Jakobsson, M., \& Wetzel, S. 2001. Security weaknesses in Bluetooth. In Cryptographers' Track at the RSA Conference (pp. 176-191). Springer Berlin Heidelberg.

[40] Ponsam, J. G., \& Srinivasan, R. 2014. A survey on MANET security challenges, attacks and its countermeasures. International Journal of Emerging Trends \& Technology in Computer Science (IJETTCS), 3(1).

[41] Sanzgiri, K., Dahill, B., Levine, B. N., Shields, C., \& Belding-Royer, E. M. 2002. A secure routing protocol for ad hoc networks. In Network Protocols, 2002. Proceedings. 10th IEEE International Conference on (pp. 78-87). IEEE.

[42] Hu, Y. C., Perrig, A., \& Johnson, D. B. 2003. Packet leashes: a defense against wormhole attacks in wireless networks. In INFOCOM 2003. TwentySecond Annual Joint Conference of the IEEE Computer and Communications. IEEE Societies (Vol. 3, pp. 1976-1986). IEEE.

[43] Borisov, N., Goldberg, I., \& Wagner, D. 2001 Intercepting mobile communications: the insecurity of 802.11. In Proceedings of the 7th annual international conference on Mobile computing and networking (pp. 180-189). ACM.

[44] Zhou, L., \& Haas, Z. J. 1999. Securing ad hoc networks. IEEE network, 13(6), 24-30.

[45] Nguyen, H. L., \& Nguyen, U. T. 2008. A study of different types of attacks on multicast in mobile ad hoc networks. Ad Hoc Networks, 6(1), 32-46.

[46] Čapkun, S., Buttyán, L., \& Hubaux, J. P. 2003. SECTOR: secure tracking of node encounters in multi-hop wireless networks. In Proceedings of the 1st ACM workshop on Security of ad hoc and sensor networks (pp. 21-32). ACM. 
[47] Hu, L., \& Evans, D. 2004. Using Directional Antennas to Prevent Wormhole Attacks. In NDSS.

[48] Mahajan, N., Bedi, R., \& Gupta, S. K. 2014. A Survey on Detection of Byzantine and Resource Consumption Attacks. Journal of Basic and Applied Engineering Research.

[49] Garg, N., \& Mahapatra, R. P. 2009. Manet security issues. IJCSNS, 9(8), 241.

[50] Guntewar, C., \& Sahare, V. 2015. A Review on Byzantine Attack Detection and Prevention Using Game Theory. (IJCSIT) International Journal of Computer Science and Information Technologies.

[51] Ishrat, Z. 2011. Security issues, challenges \& solution in MANET. IJCST, 2(4), 108-112.

[52] Boora, S., Kumar, Y., \& Kochar, B. 2011. A Survey on Security Issues in Mobile Ad-hoc Networks. IJCSMS International Journal of Computer Science and Management Studies.

[53] Singh, M., \& Kaur, G. 2013. A Surveys of Attacks in MANET. International Journal of Advanced Research in Computer Science and Software Engineering, 3(6).

[54] Lalar, S. 2014. Security in MANET: Vulnerabilities, Attacks \& Solutions. Intational J. Multidiscip. Curr. Res, 2, 62-69.

[55] Zeng, Q. A., \& Agrawal, D. P. 2002. Handbook of wireless networks and mobile computing. Publishers: John Wiley and Sons.

[56] Kaufman, C., Perlman, R., \& Speciner, M. 2002. Network security: private communication in a public world. Prentice Hall Press.

[57] Sun, J. Z. 2001. Mobile ad hoc networking: an essential technology for pervasive computing. In Info-tech and Info-net, 2001. Proceedings. ICII
2001-Beijing. 2001 International Conferences on (Vol. 3, pp. 316-321). IEEE.

[58] Gupta, V., Krishnamurthy, S., \& Faloutsos, M. 2002. Denial of service attacks at the MAC layer in wireless ad hoc networks. In MILCOM 2002 Proceedings (Vol. 2, pp. 1118-1123). IEEE.

[59] El-Hajj, W., Kountanis, D., Al-Fuqaha, A., \& Guizani, M. 2006. A fuzzy-based hierarchical energy efficient routing protocol for large scale mobile ad hoc networks (feer). In Communications, 2006. ICC'06. IEEE International Conference on (Vol. 8, pp. 3585-3590). IEEE.

[60] Achankunju, M., Pushpalakshmi, R., \& Kumar, A. V. A. 2013. Particle swarm optimization based secure QoS clustering for mobile ad hoc network. In Communications and Signal Processing (ICCSP), 2013 International Conference on (pp. 315-320). IEEE.

[61] Manisha, \& Kumar, M. 2014. Network Layer Attacks and Their Countermeasures in Manet: A Review. IOSR Journal of Computer Engineering (IOSR-JCE).

[62] Kaur, A., \& Singh, A. 2014. A Review on Security Attacks in Mobile Ad-hoc Networks. International Journal of Science and Research (IJSR), 3(5).

[63] Ghonge, M. M., Jawandhiya, P. M., \& Ali, M. S. 2011. Countermeasures of network layer attacks in manets. IJCA Special Issue on "Network Security and Cryptography" NSC.

[64] Aarti, \& Tyagi, S. S. 2013. Study Of Manet: Characteristics, challenges, application and security attacks. International Journal of Advanced Research in Computer Science and Software Engineering, 3(5),252-257. 